\title{
The Customer Is Always Right, Even When You Are Justifiably Wrong
}

C proving a business. If performed well and interpreted in the appropriate context, they are also useful vehicles for the education of staff members and for the stimulation of positive corporate change. Whether we choose to acknowledge it or not, imaging is a service industry that provides a product. That product is our opinion and advice in the form of a written report. Although our training and the traditions of western medicine may lead us to believe otherwise, in imaging the client is generally the patient's doctor rather than the patient. Through the process of referral, we are at least one step away from direct involvement in patient care, even though the information that we provide may be absolutely crucial to treatment choices and patient outcomes.

Accordingly, a survey of the opinions of referring clinicians regarding imaging reports is, in essence, an assessment of customer satisfaction. The article by Karantanis et al. in the current issue of The Journal of Nuclear Medicine (1) provides some interesting insights with respect to oncologic ${ }^{18} \mathrm{~F}$ FDG PET/CT reporting. The authors surveyed 662 clinicians from

\section{See page 1925}

a range of pertinent specialties, with the major groups being oncologists $(36.1 \%)$ and surgeons $(33.8 \%)$. There was representation from several continents, but most respondents were from Europe $(47.3 \%)$ or North America (35.0\%), and the vast majority $(85.8 \%)$ of respondents were practicing in an academic environment. Because PET/CT systems have been widely available in academic centers in both of these regions for more than a decade, one can assume a reasonably high level of experience in both the users and the providers of PET/CT services. The specified aim of the survey was to understand the frequency and nature of errors in reporting, as perceived by the end user of the information provided.

Overall, $59.3 \%$ of respondents estimated that rates of misinterpretation ranged from $5 \%$ to $20 \%$. Although this range reflects the typical accuracy reported in individual series and meta-analyses of PET/CT across various malignancies, the upper end of this range may be higher than we, as providers, would like

Received Oct. 27, 2014; accepted Oct. 28, 2014.

For correspondence or reprints contact: Rodney J. Hicks, Peter MacCallum Cancer Institute, Locked Bag 1, A'Beckett St., Victoria 8006, Australia.

E-mail: rod.hicks@petermac.org

Published online Nov. 26, 2014.

COPYRIGHT (C) 2014 by the Society of Nuclear Medicine and Molecular Imaging, Inc.

DOI: $10.2967 /$ jnumed.114.149781 to imagine is the proportion of cases in which we are incorrect. Perhaps the most instructive finding of the survey was that a significantly greater proportion of respondents believed that the major source of errors in reporting of PET/CT studies related to overinterpretation $(68.9 \%)$ rather than underinterpretation $(8.7 \%)$. This finding may come as a surprise to many expert PET/CT interpreters, who may have expected the opposite. Since the days of stand-alone ${ }^{18} \mathrm{~F}$-FDG PET, multiple causes of falsepositive results have been recognized (2). The availability of the additional information provided by the CT component should further serve to identify benign causes of focally increased glycolytic metabolism, allowing reporting clinicians to avoid interpreting benign processes as malignancy. However, the addition of CT does not overcome the intrinsic limitations of PET with respect to partial-volume effects and consequent false-negative results.

Cancer often finds diagnosticians wanting with respect to sensitivity. Its nature is to progress from a single cell, indistinguishable by many criteria from any other cell in the tissue from which it arises, to a widespread and potentially life-threatening collection of cells. This progression sometimes occurs in a relatively short period of time. Combined with a medicolegal environment that is alert to missed diagnoses and unforgiving of adverse outcomes, this knowledge provides a strong incentive toward sensitive rather than specific reporting of oncologic imaging studies. A review of old studies in cancer patients will also regularly reveal a subtle abnormality that was passed over or not commented on in a prior scan and that has subsequently progressed to being a definite lesion and highly likely to represent malignancy. In response to such "misses," we have become conditioned over time to report all such findings as disease in future studies.

Although we may intuitively reason that avoiding false-negative results is the most important objective of cancer imaging, we need to be aware that there are also significant potential harms in the overinterpretation of scans. These include invoking unnecessary patient anxiety, initiating unwarranted investigations or therapy, or potentially denying patients curative options in the mistaken belief that metastatic disease is present. A less tangible but important corollary of false-positive results is a loss of trust in even strongly positive scan results. This scenario could reduce appropriate referrals or lead to further unnecessary invasive procedures. From the perspective of our referral base, these events are more common and concerning than our fear of missing disease.

One of the defenses against being incorrect is to word reports with noncommittal phrasing. Some imaging specialists choose the option of stating that a particular diagnosis is not excluded or describe findings as being equivocal but warranting histopathologic correlation or imaging surveillance. When faced with such reports, clinicians often feel obliged to follow this advice and, more often than not, a subtle abnormality on ${ }^{18} \mathrm{~F}-\mathrm{FDG}$ PET/CT turns out to be a nonmalignant process. Although the reporting specialist's suspicion of malignancy in such cases may actually be 
very low, the referring clinician often acts on the assumption that there is at least a 50:50 chance of the lesion being cancerous.

In the study by Karantanis et al. (1), most of the respondents $(59.8 \%)$ identified the importance of greater clinical interactions by selecting "more multidisciplinary meetings with participation of both the referring and interpreting physicians" as a means to reduce the overinterpretation of results. Other popular selections included "better communication with the interpreting physician after getting the PET/CT reports" (38.4\%) and "providing a more adequate clinical history to the interpreting physician" (37.4\%). Again, these responses emphasize the importance of clinical context. I often tell my fellows that the most powerful diagnostic tool in the department is the telephone. If in doubt about how to formulate a conclusion to a report, contact the referring clinician by telephone and ask what he or she is considering and how a given result may affect treatment choices and further investigation.

Several years ago, Wang et al. reviewed how oncologists in a dedicated cancer center dealt with incidental but nonphysiologic ${ }^{18}$ F-FDG abnormalities thought not to directly relate to the diagnosis in question (3). In a consecutive series of 1,727 patients, incidental abnormalities were relatively common, being reported in 199 cases (12\%). Of 59 cases in which a second malignancy was suspected, $34(58 \%)$ were actively investigated; 14 cases had confirmed malignancies, 7 cases had unexpected metastatic sites, and 10 cases had active pathologies of potential clinical relevance to their cancer management. In most cases that were not investigated, the presence of a second malignancy was considered of low clinical importance in the context of the known malignancy. Conversely, of 122 sites presumed to be benign, only $10(8 \%)$ were actively investigated, and follow-up yielded a very low rate of malignancy. In my facility, many PET results are presented in a multidisciplinary tumor stream team meeting. My facility's experience supports the importance of communication between reporting and referring clinicians regarding the likely gravity of ${ }^{18}$ F-FDG PET/CT findings and the importance of considering imaging findings in a broader clinical context.

To err is human, but it is also our nature to never want to admit that we err. This concept is reflected in the irony of my father's favorite saying, "I'm always right. I thought I was wrong once, but I was mistaken." In this context, we should consider the possibility that the perceptions of referring clinicians may be wrong and that we are right much more often than they give us credit for. Surgeons, in particular, are greatly influenced by pathologic reports, particularly if no disease is identified at a site of abnormality. In over $20 \mathrm{y}$ of reporting ${ }^{18} \mathrm{~F}$-FDG PET scans, I have encountered many cases in which an apparently false-positive result was conveyed to me on the basis of a negative biopsy but the patient subsequently relapsed or showed progression at the very site of an abnormality, often with a surgical clip right beside it! In such cases, the patient often ended up in the care of an oncologist rather than the surgeon, who remained blissfully ignorant that the problem was a pathologic sampling error rather than a false-positive imaging finding. Accordingly, I have a picture on my wall that advises clinicians who remark on a "false-positive" result without a cogent, alternative pathologic process to explain the observation to "watch this space."

\section{DISCLOSURE}

No potential conflict of interest relevant to this article was reported.

Rodney J. Hicks

The University of Melbourne

East Melbourne, Victoria, Australia

\section{REFERENCES}

1. Karantanis D, Kalkanis D, Czernin J, et al. Perceived misinterpretation rates in oncologic ${ }^{18}$ F-FDG PET/CT studies: a survey of referring physicians. J Nucl Med. 2014;55:1925-1929.

2. Shreve PD, Anzai Y, Wahl RL. Pitfalls in oncologic diagnosis with FDG PET imaging: physiologic and benign variants. Radiographics. 1999;19:61-77.

3. Wang G, Lau EW, Shakher R, et al. How do oncologists deal with incidental abnormalities on whole-body fluorine-18 fluorodeoxyglucose PET/CT? Cancer. 2007;109:117-124. 